\title{
Full Duplex Relaying in Non Orthogonal Multiple Access System with Advanced Successive Interference Cancellation
}

\author{
Qaiser HUSSAIN ${ }^{1}$, Sarmad SOHAIB ${ }^{1,2}$ \\ ${ }^{1}$ Department of Electrical Engineering, University of Engineering and Technology, Taxila 47050, Pakistan \\ ${ }^{2}$ Department of Electrical and Electronic Engineering, University of Jeddah, KSA \\ qaiser_awan78@hotmail.com,ssohaib@uj.edu.sa \\ Submitted April 14, 2020 / Accepted September 7, 2020
}

\begin{abstract}
This paper describes a full-duplex (FD) cooperative non orthogonal multiple access (NOMA) system with dedicated relay under residual self-interference (RSI). An advanced successive interference cancellation (ASIC) technique is proposed in the FD cooperative NOMA system as an alternate of successive interference cancellation (SIC) scheme. The ASIC scheme maps the received signal into subgroups and by applying conventional SIC scheme on each subgroup results in separation of signals. The approximated analytical expressions of outage probability and ergodic sum rate for proposed ASIC based FD DF cooperative NOMA system are derived and the system throughput is analysed. Finally, according to the results, our proposed ASIC based FD DF cooperative NOMA system shows better outage performance and higher ergodic sum rate as compared to conventional SIC based FD DF cooperative NOMA system.
\end{abstract}

\section{Keywords}

Advanced successive interference cancellation, full duplex, non orthogonal multiple access, residual selfinterference

\section{Introduction}

Non orthogonal multiple access (NOMA) is considered as an attractive solution to achieve high spectral efficiency, large connectivity and low latency as compared to orthogonal multiple access (OMA) technique. The performance gains of NOMA system are assumed as the main requirement for future generation wireless communication systems [1-3]. Several NOMA solutions are deeply studied in [4], [5]. NOMA schemes are actively divided into two types; power domain NOMA [6], and code domain NOMA [7]. According to power domain NOMA, the signals of different users communicating to same Base Station (BS) are combined by allocating different power levels and information signal components of composite signal are separated by applying Successive Interference Cancellation (SIC) technique [8], [9]. Power allocation is done based on channel gain of different users i.e. allocation of more power is reserved for low channel gain users and vice versa [9]. Power domain NOMA is useful in both uplink and downlink scenarios, however, its application are more in downlink [10], [11]. Uplink NOMA is investigated to achieve high system capacity and the new approximated expressions for outage probability and ergodic sum rate are derived in [11], [12], whereas downlink NOMA systems are extensively studied to evaluate outage probability and sum rates in [13]. The authors in [14] have introduced a novel concept of cognitive radio inspired NOMA system where the fixed power allocation in NOMA systems was discussed by using the influence of user pairing. In this paper [15], the authors have proposed a Simplified Collaborated NOMA Assisted Relaying (S-CNAR) system for 5G that reduces the problem of relay complexity for CNAR system. The outage probabilities and ergodic sum capacity of both systems are analysed and the S-CNAR system attains the same performance similar to CNAR system but with much lower complexity. The author in [16] has proposed an Advanced Successive Interference Cancellation (ASIC) technique as an alternate of SIC technique for NOMA systems, where the problem of user delay during decoding process due to iterative decoding nature of SIC is resolved.

Cooperative NOMA is a very useful and active area among various research directions where the transmission reliability of the system is enhanced by using dedicated relays or user relay nodes. In [17], [18], the authors have elaborated the function of dedicated relay to receive message from source and then decodes and forwards it to destination. The function of relay based on user cooperation was first discussed in [19], where the users experiencing bad channel conditions take the benefits of users with good channel conditions. Aforementioned research contributions use Half-Duplex (HD) relay resulting in improved transmission reliability at the cost of reduced spectral efficiency due to usage of additional channel resources. One of the solutions to the problem of spectral effi- 
ciency loss in half duplex relaying scheme is the Full-Duplex (FD) relay technology that has attained significant research attention due to the advancement of Self-Interference (SI) cancellation techniques. These SI cancellation techniques cannot completely remove SI at FD relays but remains some SI in the system, which is called Residual Self-Interference (RSI) [20], [21]. We have assumed to model RSI as an additive white Gaussian signal with zero mean and variance proportional to transmit relay power [22]. The author in [23] discussed the performance of FD Amplify-and-Forward (AF) relaying by assuming no direct link between source and destination under RSI, while outage performance of FD DF relaying system is deeply elaborated in [24]. The performance of FD Compress-and-Forward (CF) relaying system is investigated under RSI in [25] where the author has emphasized that the full transmit power at relay is not always useful for the system and the maximum achievable rates are obtained at optimum relay transmit power.

The significant capacity enhancements of future wireless communication networks are obtained by integrating NOMA systems and FD relay technology. In [26], the outage performance of cooperative NOMA system based on FD device to device scheme is studied and investigated. The performance of the downlink cooperative NOMA system is briefly studied over independent identically distributed (i.i.d) Rayleigh fading channels where the approximated closed form expressions of outage probability, ergodic sum rate and asymptotic analysis are evaluated to show that the NOMA gives better performance as compared to OMA by improving spectral efficiency and user fairness [27]. The outage probability and ergodic sum rate of FD DF cooperative NOMA system are studied and evaluated under RSI in [28].

The improvement in transmission reliability of these cooperative NOMA systems is obtained at the cost of reduced spectral efficiency due to user delay decoding process caused by SIC at user end. In this paper, we propose ASIC in place of SIC at FD DF relay node of cooperative NOMA system. No direct link is assumed between BS and NOMA users in our proposed system. The proposed ASIC based FD DF cooperative NOMA system outperforms the conventional SIC based FD DF cooperative NOMA system. The question that remains is how much spectral efficiency and outage performance can be improved. In order to answer this important question, the scenario is kept same as discussed in [28]. The main contributions of this paper are summarised as:

- The proposed ASIC based FD DF cooperative NOMA system is analysed and discussed by calculating approximated expressions of outage probability under the assumption of no direct link between BS and far users.

- The proposed ASIC based FD DF cooperative NOMA system is also evaluated by using closed form approximations of ergodic achievable rates and ergodic sum rate.

- The proposed ASIC based FD DF cooperative NOMA system is compared with the traditional SIC based FD DF cooperative NOMA system under RSI.

- The proposed ASIC based FD DF cooperative NOMA system shows better outage performance and higher ergodic sum rate than traditional SIC based FD DF cooperative NOMA system in medium to high SNR region under RSI. The proposed scheme provides higher system throughput under the same conditions.

The organization of rest of paper is given as follows. The system model of ASIC based FD DF cooperative NOMA system with dedicated relay is described in Sec. 2. Outage probability and ergodic sum rate of the proposed scheme are derived in Sec. 3. The comparison of results for the proposed system and the conventional system are explained in Sec. 4. Finally, Section 5 concludes the paper.

\section{System Model}

Figure 1 illustrates the system model where the Base Station (BS) intends to communicate with the far users $D_{1}$, $D_{2}$ and $D_{3}$ by using dedicated relay $R$ that works in FD DF mode. Two antennas are installed on $R$ for it to operate in FD mode, whereas the remaining nodes employ single antenna. Furthermore, due to shadowing, no direct link is assumed between BS and far users. Hereafter, we use subscripts s, r, 1, 2, and 3 for BS, relay, far user 1, 2 and 3, respectively. All wireless channels are considered as independent block Rayleigh fading channels. The $i \rightarrow j$ link is represented as the channel coefficient $h_{i j}$, where $i j \in\{s r, r 1, r 2, r 3\}$. Therefore, $h_{i j}=g_{i j} d_{i j}{ }^{-\alpha}$ and $g_{i j} \sim \operatorname{CN}\left(0, \lambda_{i j}{ }^{\mathrm{g}}\right) . d_{i j}{ }^{-\alpha}$ represents the path loss, where $d_{i j}$ represents the distance between $i$ and $j$ nodes and $\alpha$ is path loss exponent. The channel gain $\left|g_{i j}\right|^{2}$ is considered as independent exponential distributed random variable with mean $\rho_{i j}{ }^{\mathrm{g}}$. It is assumed that the transmission power for the source and relay are same, i.e. $P_{\mathrm{s}}=P_{\mathrm{r}}=P$. Noise is assumed to be additive white Gaussian noise (AWGN) with zero mean and $N_{0}$ variance unless otherwise stated.

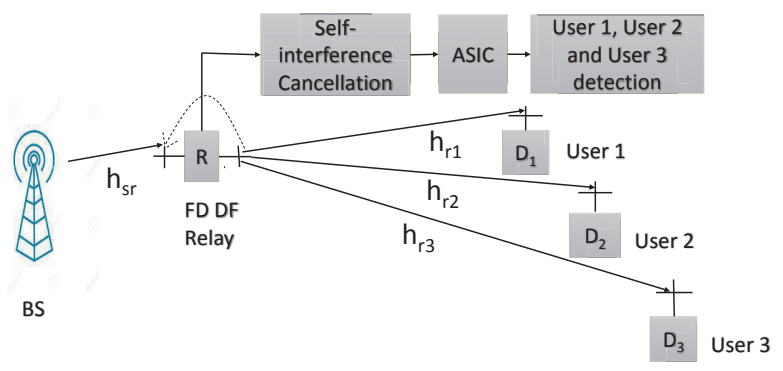

Fig. 1. Cooperative NOMA with full duplex relaying system. 
The receiver SNR is defined as $\gamma_{i j}=\frac{P\left|h_{i j}\right|^{2}}{N_{0}}$ which is considered as exponentially distributed random variable with mean $\rho_{i j}{ }^{\gamma}=\frac{\lambda_{i j}{ }^{\mathrm{g}} P d_{i j}{ }^{-2 \alpha}}{N_{0}}$.

During the $k$-th time slot, BS transmits the superimposed signal to far users given as [13]

$$
s[k]=\left(\sqrt{a_{1} P_{\mathrm{s}}} x_{1}[k]+\sqrt{a_{2} P_{\mathrm{s}}} x_{2}[k]+\sqrt{a_{3} P_{\mathrm{s}}} x_{3}[k]\right)
$$

where $x_{i}$ and $a_{i}$ denote the information symbols and the power allocation coefficients for users $i$, such that $a_{1}+a_{2}+$ $a_{3}=1$ and $a_{1}<a_{2}<a_{3}$. Due to FD DF relay in the system, it receives the composite signal and RSI signal simultaneously. Therefore, the signal received at relay is expressed as [28]

$$
y_{\mathrm{r}}[k]=h_{\mathrm{sr}} s[k]+v[k]+n_{\mathrm{r}}[k]
$$

where $v(k)$ represents the RSI due to FD operation at $R$. The RSI is also modelled as AWGN signal with zero mean and variance is given by

$$
V=\beta P_{\mathrm{r}}^{\Gamma}
$$

where $\beta$ and $\Gamma$ are considered as constants which are the function of self-interference cancellation techniques. The variance model in (3) clearly shows that the RSI increases with $P_{\mathrm{r}}[25]$.

The received signal at $R$ is mapped into subgroups according to ASIC scheme. Here the number of information elements in the received signal is equal to 3 and $a_{1}<a_{2}<a_{3}$. The received signal is mapped as [16]

$$
y_{\mathrm{r}}[k]=y_{\mathrm{ra}_{\mathrm{a}}}[k]+y_{\mathrm{rb}}[k]
$$

where

$y_{\mathrm{ra}}[k]=h_{\mathrm{sr}}\left(\sqrt{a_{1} P_{\mathrm{s}}} x_{1}[k]+\sqrt{a_{3} P_{\mathrm{s}}} x_{3}[k]\right)+v_{1}[k]+n_{\mathrm{ra}}[k]$ and

$$
y_{\mathrm{rb}}[k]=\sqrt{a_{2} P_{\mathrm{s}}} h_{\mathrm{sr}} x_{2}[k]+v_{2}[k]+n_{\mathrm{rb}}[k]
$$

where $v_{1}[k]$ and $v_{2}[k]$ are $\mathcal{C N}(0, V / 2)$, and $n_{\mathrm{ra}}[k]$ and $n_{\mathrm{rb}}[k]$ are $C \mathcal{N}\left(0, N_{0} / 2\right)$. The construction process of the number of subgroups is simply based on number of signal components and system configuration. Applying conventional SIC technique on each subgroup results in detection of the desired signal.

The information signals for users $D_{1}, D_{2}$ and $D_{3}$ can be successfully decoded at $R$. Therefore, the Signal to Interference and Noise Ratio (SINR) for user $D_{3}$ at $R$ is written as

$$
\begin{aligned}
\gamma_{\mathrm{r}, 3} & =\frac{a_{3} P\left|h_{\mathrm{sr}}\right|^{2}}{a_{1} P\left|h_{\mathrm{sr}}\right|^{2}+\frac{V}{2}+\frac{N_{0}}{2}} \\
& =\frac{a_{3} \gamma_{\mathrm{sr}}}{a_{1} \gamma_{\mathrm{sr}}+\frac{V}{2 N_{0}}+\frac{1}{2}}
\end{aligned}
$$

where $\gamma_{\mathrm{sr}}=\frac{P\left|h_{\mathrm{sr}}\right|^{2}}{N_{0}}$. The user $D_{3}$ message is decoded at relay $R$ and its rate is given by [28]

$$
C_{\mathrm{r}, 3}=\log _{2}\left(1+\gamma_{\mathrm{r}, 3}\right) .
$$

Now, the effective SINR of user $D_{1}$ message after performing $\mathrm{SIC}$ is given as

$$
\gamma_{\mathrm{r}, 1}=\frac{a_{1} \gamma_{\mathrm{sr}}}{\frac{V}{2 N_{0}}+\frac{1}{2}} .
$$

User $D_{1}$ 's achievable rate can be calculated as

$$
C_{\mathrm{r}, 1}=\log _{2}\left(1+\gamma_{\mathrm{r}, 1}\right) \text {. }
$$

The effective SINR of user $D_{2}$ message at $R$ is given by

$$
\gamma_{\mathrm{r}, 2}=\frac{a_{2} \gamma_{\mathrm{sr}}}{\frac{V}{2 N_{0}}+\frac{1}{2}}
$$

and, the rate of user $D_{2}$ at $R$ is given by

$$
C_{\mathrm{r}, 2}=\log _{2}\left(1+\gamma_{\mathrm{r}, 2}\right) \text {. }
$$

The decoded signals $\tilde{x}_{1}, \tilde{x}_{2}$ and $\tilde{x}_{3}$ at $R$ are broadcasted to far users i.e. from $R$ to $D_{1}, D_{2}$, and $D_{3}$. The user $D_{1}$ receives its signal as

$$
y_{D_{1}}[k]=h_{\mathrm{r} 1} z[k]+n_{1}[k]
$$

where

$z[k]=\left(\sqrt{c_{1} P_{\mathrm{r}}} \tilde{x}_{1}[k-\delta]+\sqrt{c_{2} P_{\mathrm{r}}} \tilde{x}_{2}[k-\delta]+\sqrt{c_{3} P_{\mathrm{r}}} \tilde{x}_{3}[k-\delta]\right)$

where $\delta>1$ is the processing delay and the power allocation coefficients for decoded users 1,2 , and user 3 are set as $c_{1}$, $c_{2}$ and $c_{3}$, such that $c_{1}+c_{2}+c_{3}=1$ and $c_{1}<c_{2}<c_{3}$. The message of user $D_{3}$ decoded at user $D_{1}$ has the achievable rate given as

$$
C_{1,3}=\log _{2}\left(1+\gamma_{1,3}\right)
$$

where $\gamma_{1,3}=\frac{c_{3} \gamma_{\mathrm{r} 1}}{c_{1} \gamma_{\mathrm{r} 1}+\frac{1}{2}}, \gamma_{1,1}=\frac{c_{1} P\left|h_{\mathrm{r} 1}\right|^{2}}{\frac{N_{0}}{2}}=2 c_{1} \gamma_{\mathrm{r} 1}$ are the effective SINRs of users $D_{3}$ and $D_{1}$ observed at user $D_{1}$ and $\gamma_{\mathrm{r} 1}=\frac{P\left|h_{\mathrm{r} 1}\right|^{2}}{N_{0}}$. The rate of user $D_{1}$ at $D_{1}$ is given as

$$
C_{1,1}=\log _{2}\left(1+\gamma_{1,1}\right) \text {. }
$$

Similarly, the signal received by user $D_{2}$ is expressed by

$$
y_{D_{2}}[k]=h_{\mathrm{r} 2} z[k]+n_{2}[k],
$$

the rate of user $D_{2}$ at $D_{2}$ is

$$
C_{2,2}=\log _{2}\left(1+\gamma_{2,2}\right)
$$

where $\gamma_{2,2}=2 c_{2} \gamma_{\mathrm{r} 2}$ is the effective SINR of user $D_{2}$ and $\gamma_{\mathrm{r} 2}=\frac{P\left|h_{\mathrm{r} 2}\right|^{2}}{N_{0}}$.

Finally, the user $D_{3}$ receives its signal as

$$
y_{D_{3}}[k]=h_{\mathrm{r} 3} z[k]+n_{3}[k],
$$

hence, the rate of user $D_{3}$ can be calculated as

$$
C_{3,3}=\log _{2}\left(1+\gamma_{3,3}\right)
$$


where $\gamma_{3,3}=\frac{c_{3} \gamma_{\mathrm{r} 3}}{c_{1} \gamma_{\mathrm{r} 3}+\frac{1}{2}}$ is the effective SINR of user $D_{3}$ and $\gamma_{\mathrm{r} 3}=\frac{P\left|h_{\mathrm{r} 3}\right|^{2}}{N_{0}}$.

Since the maximum achievable rate is resulted from weakest link as observed in Fig. 1. Therefore, the maximum achievable rate for user $D_{1}$ is [28]

$$
C_{1}=\log _{2}\left(1+\min \left\{\gamma_{\mathrm{r}, 1}, \gamma_{1,1}\right\}\right) \text {. }
$$

Similarly, the far user $D_{2}$ can compute its maximum achievable rate as

$$
C_{2}=\log _{2}\left(1+\min \left\{\gamma_{\mathrm{r}, 2}, \gamma_{2,2}\right\}\right) .
$$

Finally, since $\tilde{x}_{3}[k-\delta]$ is also decoded at $D_{1}$ for ASIC, therefore the maximum achievable rate of user $D_{3}$ is calculated as

$$
C_{3}=\log _{2}\left(1+\min \left\{\gamma_{\mathrm{r}, 3}, \gamma_{1,3}, \gamma_{3,3}\right\}\right) .
$$

\section{Performance Analysis}

The proposed ASIC based FD DF cooperative NOMA system is evaluated in this section by using outage probability and ergodic sum rate.

\subsection{Outage Probability}

The ASIC based FD DF cooperative NOMA system is analysed in terms of outage probability in this subsection. The targeted threshold values for SINR of NOMA users are represented by $\gamma_{\mathrm{th} 1}=2^{R_{1}}-1, \gamma_{\mathrm{th} 2}=2^{R_{2}}-1$ and $\gamma_{\mathrm{th} 3}=2^{R_{3}}-1$, where $R_{1}, R_{2}$ and $R_{3}$ are corresponding threshold targeted rates of users $D_{1}, D_{2}$, and $D_{3}$ respectively.

The outage probability of far user $D_{1}$ is written as

$$
\begin{aligned}
P_{D 1}^{F D} & =1-P\left(C_{\mathrm{r}, 1}>R_{1} C_{1,1}>R_{1} C_{1,3}>R_{3}\right) \\
& =1-\mathrm{e}^{-\theta_{1}}
\end{aligned}
$$

where $\theta_{1}=\left[\frac{\gamma_{\mathrm{th} 1}\left(V+N_{0}\right)}{2 a_{1} \rho_{\mathrm{sr}}^{\gamma}}+\frac{\gamma_{\mathrm{th} 1}}{2 c_{1} \rho_{\mathrm{r} 1} \gamma}+\frac{\gamma_{\mathrm{th} 3}}{2\left(c_{3}-c_{1} \gamma_{\mathrm{th} 3}\right) \rho_{\mathrm{r} 1} \gamma}\right]$.

The proof is given in appendix A.

The outage probability of far user $D_{2}$ is

$$
\begin{aligned}
P_{D 2}^{F D} & =1-P\left(C_{\mathrm{r}, 2}>R_{2} \quad C_{2,2}>R_{2}\right) \\
& =1-\mathrm{e}^{-\frac{\gamma_{\mathrm{th} 2} \theta_{2}}{2}}
\end{aligned}
$$

where $\theta_{2}=\left(\phi_{1}+\phi_{2}\right), \phi_{1}=\frac{1}{c_{2} \rho_{\mathrm{r} 2} \gamma}$, and $\phi_{2}=\frac{V+N_{0}}{a_{2} \rho_{\mathrm{sr}}{ }^{\gamma}}$.

The proof is given in appendix $\mathrm{B}$.

The outage probability of far user $D_{3}$ is

$$
\begin{aligned}
P_{D 3}^{F D} & =1-P\left(C_{\mathrm{r}, 3}>R_{3} \quad C_{3,3}>R_{3}\right) \\
& =1-\mathrm{e}^{-\frac{\gamma_{\mathrm{th} 3} \theta_{3}}{2}}
\end{aligned}
$$

where $\theta_{3}=\left(\psi_{1}+\psi_{2}\right), \psi_{1}=\frac{1}{\left(c_{3}-c_{1} \gamma_{\mathrm{th} 3}\right) \rho_{\mathrm{r} 3} \gamma}$, and $\psi_{2}=$ $V+N_{0}$ $\overline{\left(a_{3}-a_{1} \gamma_{\mathrm{th} 3}\right) \rho_{\mathrm{sr}} \gamma}$

The proof is given in appendix $\mathrm{C}$.

The expression of throughput for proposed ASIC based FD DF NOMA system is evaluated by using results of outage probabilities of (21), (22) and (23). Therefore, the system throughput for our proposed system without direct link is

$$
\begin{aligned}
R_{\mathrm{T}}^{F D} & =\left(1-P_{\mathrm{D} 1}^{F D}\right) R_{1}+\left(1-P_{\mathrm{D} 2}^{F D}\right) R_{2} \\
& +\left(1-P_{\mathrm{D} 3}^{F D}\right) R_{3} .
\end{aligned}
$$

\subsection{Ergodic Rate}

The expected values of achievable rates for users 1, 2, and 3 are known as ergodic rates which are derived in this section.

The ergodic rate of user $D_{1}$ is calculated as

$$
R_{D 1}^{F D}=\frac{\mathrm{e}^{\left(\frac{V+1}{2 a_{1} \rho_{\mathrm{sr}} \gamma}+\frac{1}{2 c_{1} \rho_{\mathrm{r} 1} \gamma}\right)}}{\ln 2} \times \mathrm{E}_{1}\left(\frac{V+1}{2 a_{1} \rho_{\mathrm{sr}} \gamma}+\frac{1}{2 c_{1} \rho_{\mathrm{r} 1} \gamma}\right)
$$

where, $\mathrm{E}_{1}(x)=\int_{x}^{\infty} \frac{\mathrm{e}^{-u}}{u} \mathrm{~d} u$, represents definition of exponential integral function and used in the above equation.

The proof is given in appendix D.

The far user $D_{2}$ evaluates its ergodic rate according to the relation given as

$$
\begin{aligned}
R_{\mathrm{D} 2}^{F D} & =\frac{\mathrm{e}^{\left(\frac{V+1}{2 a_{2} \rho_{\mathrm{sr}} \gamma}+\frac{1}{2 c_{2} \rho_{\mathrm{r} 2} \gamma}\right)}}{\ln 2} \\
& \times\left[\mathrm{E}_{1}\left(\left(\frac{V+1}{2 a_{2} \rho_{\mathrm{sr}} \gamma}+\frac{1}{2 c_{2} \rho_{\mathrm{r} 2} \gamma}\right)\right] .\right.
\end{aligned}
$$

The proof is given in appendix E.

The ergodic rate of far user $D_{3}$ for proposed FD DF NOMA system can be approximated by using Gaussian quadrature method. The ergodic rate of user $D_{3}$ is calculated as

$$
R_{D 3}^{F D}=\frac{a_{3}}{2 a_{1} \ln 2} \times \sum_{i=1}^{N} w_{i} g\left(\frac{a_{3}}{2 a_{1}}\left(X_{i}+1\right)\right)
$$

where $w_{i}$ and $X_{i}$ are the weights and arguments of the function and $g(x)$ has been given in (F5).

The proof is given in appendix $\mathrm{F}$.

By using (25), (26) and (27), the ergodic sum rate of the proposed FD DF cooperative NOMA system is expressed as

$$
R_{D T}^{F D}=R_{D 1}^{F D}+R_{D 2}^{F D}+R_{D 3}^{F D}
$$




\section{Results and Discussions}

The proposed ASIC based FD DF cooperative NOMA system is compared with traditional SIC based FD DF cooperative NOMA system by using performance parameters of outage probability and ergodic sum rate in this section. MATLAB (R2014a) programming environment is used to plot the result. ${ }^{1}$ To validate our analytical results, the parameter values used in the results are given in Tab. 1 .

\begin{tabular}{|c|c|}
\hline Parameters & Values \\
\hline Path loss exponent: $\alpha$ & 2 \\
\hline Source to relay distance: $d_{\mathrm{sr}}$ & $3 m$ \\
\hline Relay to user 1 distance: $d_{\mathrm{r} 1}$ & $5 m$ \\
\hline Relay to user 2 distance: $d_{\mathrm{r} 2}$ & $7 \mathrm{~m}$ \\
\hline Relay to user 3 distance: $d_{\mathrm{r} 3}$ & $9 m$ \\
\hline Mean of channel gain $\left|g_{\mathrm{sr}}\right|^{2}: \rho_{\mathrm{sr}}{ }^{g}$ & 1 \\
\hline Mean of channel gain $\left|g_{\mathrm{r} 1}\right|^{2}: \rho_{\mathrm{r} 1}{ }^{g}$ & 1 \\
\hline Mean of channel gain $\left|g_{\mathrm{r} 2}\right|^{2}: \rho_{\mathrm{r} 2}{ }^{g}$ & 1 \\
\hline Mean of channel gain $\left|g_{\mathrm{r} 3}\right|^{2}: \rho_{\mathrm{r} 3}{ }^{g}$ & 1 \\
\hline Threshold targeted rates: $R_{1} / R_{2} / R_{3}$ & $3 / 2 / 1.5$ \\
\hline Power allocation coefficients: $a_{1} / a_{2} / a_{3}$ & $0.05 / 0.2 / 0.75$ \\
\hline
\end{tabular}

Tab. 1. The name of parameters (variables).

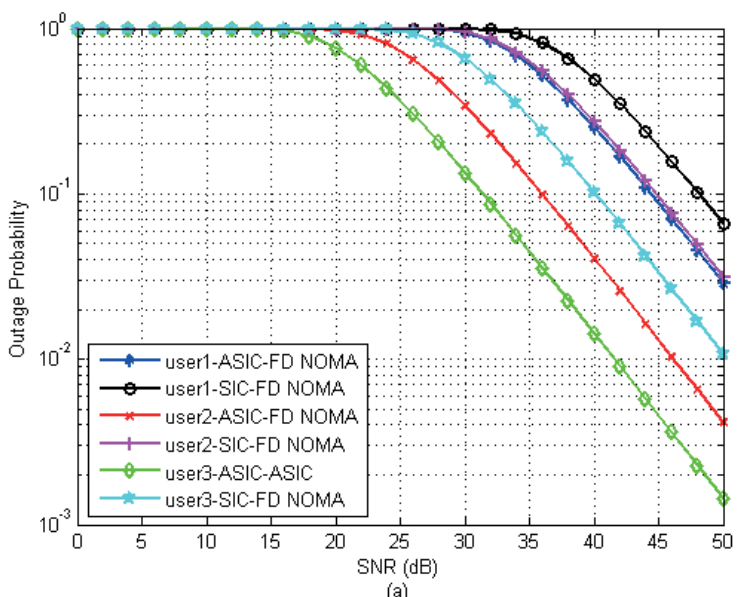

(a)

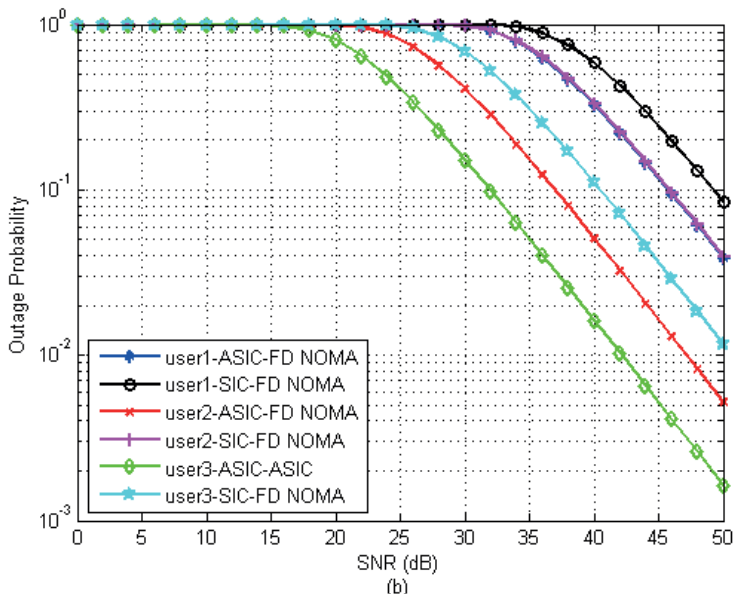

Fig. 2. Outage probability vs transmit SNR with (a) $\beta=0$, (b) $\beta=0.9$.

\footnotetext{
${ }^{1} \mathrm{~A}$ copy of the corresponding MATLAB model is available upon request at qaiser_awan78@hotmail.com.
}

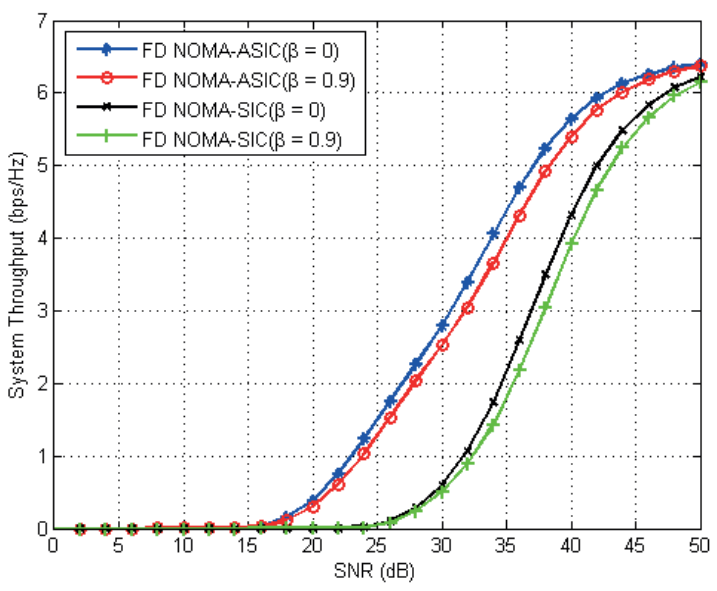

Fig. 3. Throughput vs SNR for various levels of RSI.

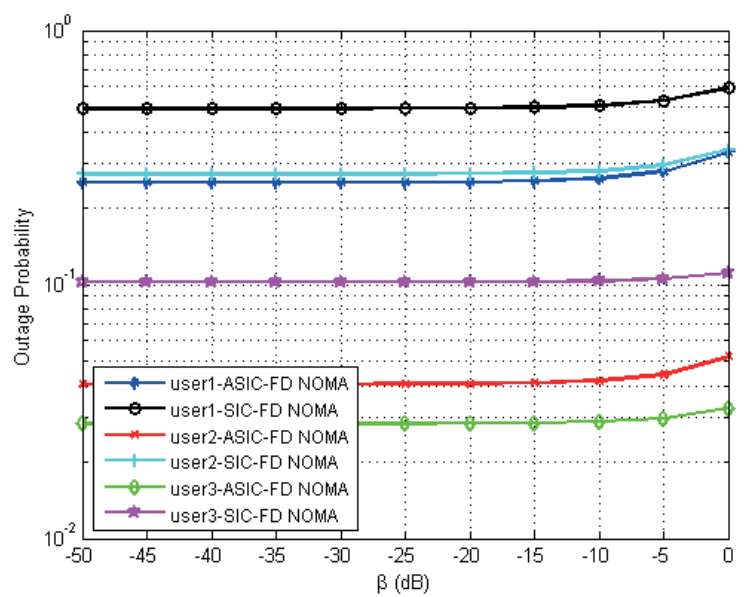

Fig. 4. Outage probability vs $\beta$ for $\mathrm{SNR}=40 \mathrm{~dB}$.

Figure 2 shows the outage probability for proposed ASIC based FD DF cooperative NOMA system and traditional SIC based FD DF cooperative NOMA system versus transmit SNR with (a) $\beta=0$ and (b) $\beta=0.9$. The analytical curves of outage probabilities for all three NOMA users are plotted according to (21), (22), and (23). It is shown that by comparing outage performance of the proposed ASIC based FD NOMA system and traditional SIC based FD NOMA system under different levels of RSI, the former system gives better performance. Due to more outage probability of user 1 than other two users, user 1 decodes the messages of other two users by performing ASIC in the corresponding FD cooperative NOMA system. However, user 3 has low outage probability than users 1 , and 2 because it directly decodes its message. Figure 2 (a) clearly shows that outage performance of the proposed ASIC based FD DF cooperative NOMA system is better than traditional SIC based FD DF cooperative NOMA system in the SNR (dB) range of [29,50], $[21,50]$, and $[17,50]$ for users 1,2 , and 3 respectively under 
no $\operatorname{RSI}(\beta=0)$. The situation is also similar in Fig. 2 (b) but for SNR $(\mathrm{dB})$ range of $[31,50],[22.5,50]$, and [17.5,50] with RSI level $(\beta=0.9)$. Therefore, the proposed ASIC scheme outperforms the traditional SIC scheme used in cooperative NOMA systems [26-28]. In Fig. 3, we compare the throughput for proposed ASIC based FD DF NOMA system and traditional SIC based FD DF NOMA system with different values of $\beta$. The system throughput is obtained by plotting the solid curves of proposed ASIC based FD NOMA system according to (24). It is observed from figure that the proposed ASIC based FD NOMA system achieves higher throughput than traditional SIC based FD NOMA system under different levels of RSI. It is also seen that the increase of RSI levels (i.e. $\beta=0$ to $\beta=0.9$ ) result the reduction of system throughput for both systems. Both systems attain same throughput in low and high SNR range, whereas performance gap between them becomes larger in moderate SNR range.

Figure 4 plots the comparison of outage probability against various values of $\beta$ for proposed ASIC based FD DF cooperative NOMA system and conventional SIC based FD DF cooperative NOMA system. In both systems, the relay functions in FD mode. It is clearly shown in Fig. 4 that the outage behaviour of both systems does not change significantly for low values of RSI (i.e. $\beta=-50 \mathrm{~dB}$ to $\beta \approx$ $-10 \mathrm{~dB})$. The outage performance of both systems starts to change for high values of RSI (i.e. $\beta=-10 \mathrm{~dB}$ to $\beta \approx$ $-1 \mathrm{~dB})$. However, the outage performance of our proposed system remains higher than traditional system under various levels of RSI. Finally, the performance of our proposed system becomes much better than traditional system under no self-interference cancellation at relay (i.e. $\beta=0$ ). These results indicate the superiority of outage performance for our proposed system than traditional system under different levels of RSI.

Figure 5 represents the ergodic rates of NOMA users against transmit SNR for proposed ASIC based FD cooperative NOMA system and traditional SIC based FD cooperative NOMA system with (a) $\beta=0$ and (b) $\beta=0.9$. As shown in figure, the analytical formulas for ergodic rates of user 1 , user 2, and user 3 represented by (25), (26), and (27) respectively are plotted. It can be observed that the ergodic rate of all users for both systems increases with increasing transmit SNR in the low to moderate SNR range. However, the ergodic rate of user 3 for both systems almost stops increasing at above $37 \mathrm{~dB}$ of transmit SNR and attains the fixed value. The ergodic rate of NOMA users for our proposed system becomes higher than that of traditional system at above 18 $\mathrm{dB}$ of transmit SNR as shown in Fig. 5 (a). The similar situation occurs in Fig. 5 (b) but at the transmit SNR of above 20 $\mathrm{dB}$. Therefore, it is shown that proposed ASIC based FD DF cooperative NOMA system has higher ergodic rates than traditional SIC based FD DF cooperative NOMA system from moderate to high SNR region for no RSI $(\beta=0)$ and RSI level $(\beta=0.9)$.
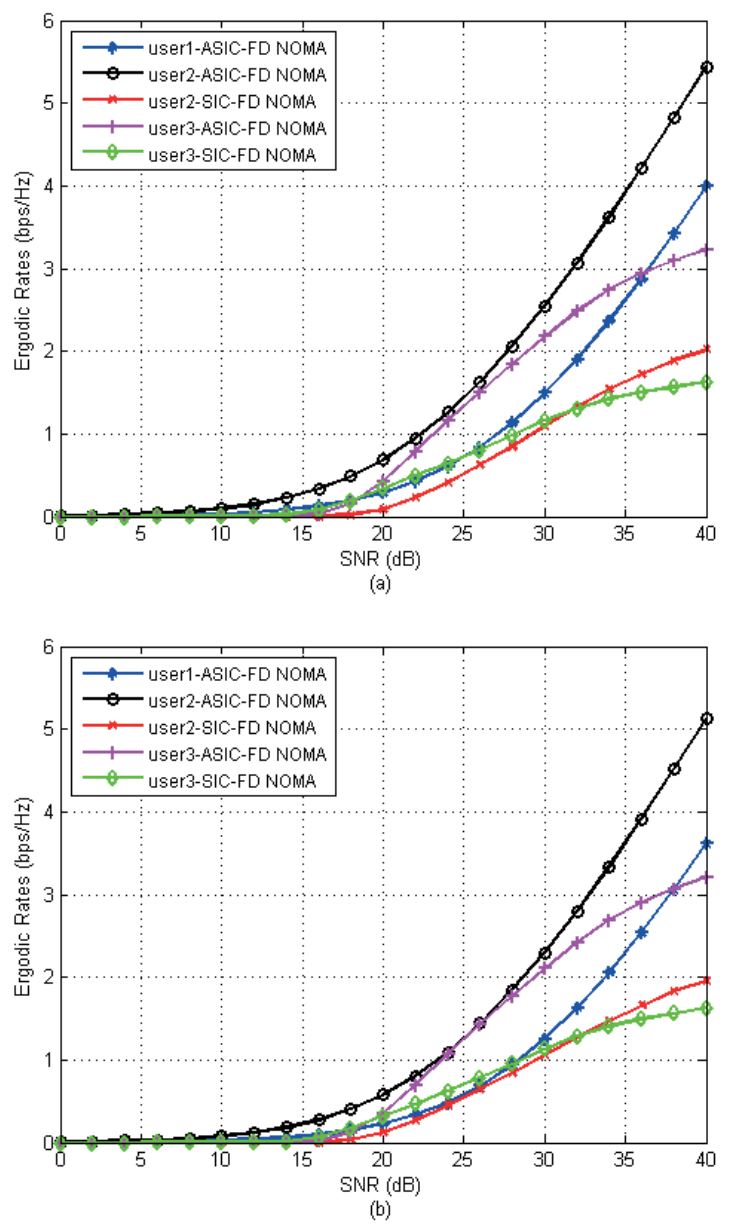

Fig. 5. Ergodic achievable rates vs the transmit SNR with (a) $\beta=0$, (b) $\beta=0.9$.

Figure 6 represents the variations of ergodic sum rate for proposed ASIC based FD cooperative NOMA system and traditional SIC based FD cooperative NOMA system versus transmit SNR with various levels of RSI.

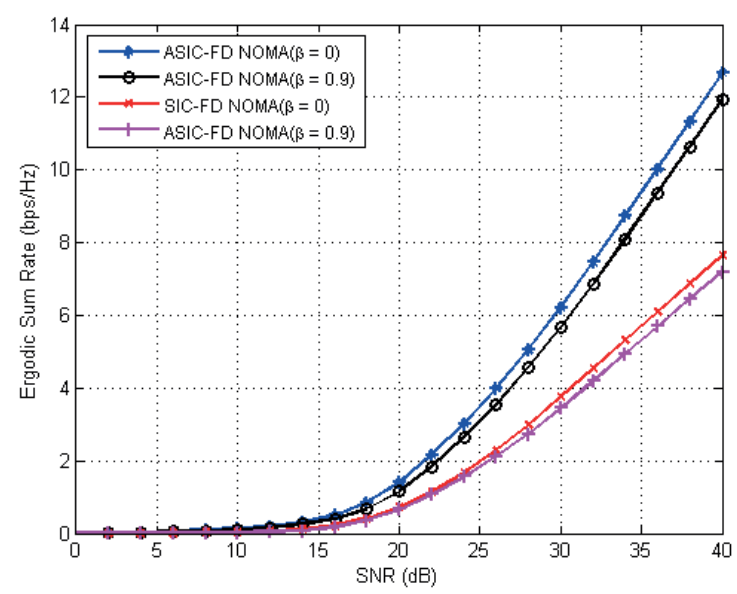

Fig. 6. Ergodic sum rate vs transmit SNR for different levels of RSI. 


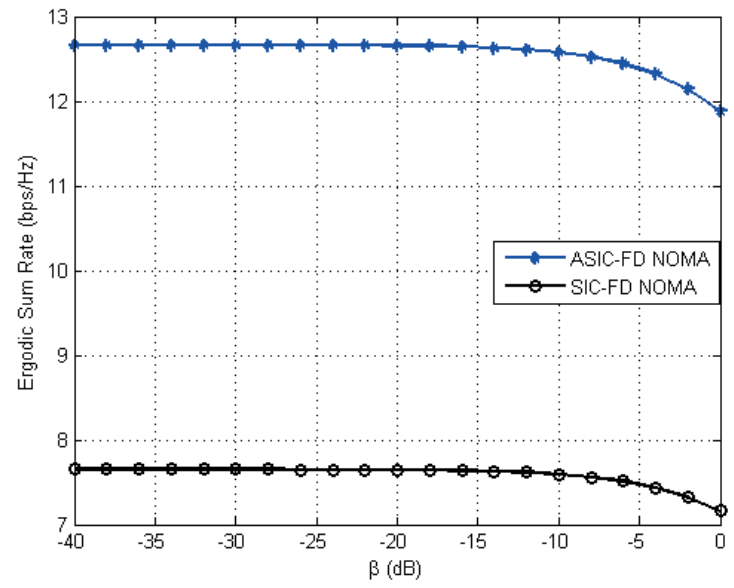

Fig. 7. Ergodic sum rate vs $\beta$ for $\mathrm{SNR}=40 \mathrm{~dB}$.

The ergodic sum rate of the proposed system is obtained by plotting the solid curves according to (28). The figure indicates that the ergodic sum rate of the proposed ASIC based FD NOMA system becomes higher than traditional SIC based FD NOMA system at about above $14 \mathrm{~dB}$ of transmit SNR. It is also seen that the ergodic sum rate for both systems decreases with the increase of RSI levels (i.e. by setting $\beta=0$ to $\beta=0.9$ ). However, it is observed that the ergodic sum rate of our proposed system is still higher than traditional system under these different levels of RSI.

Figure 7 represents the comparison of the ergodic sum rate for proposed system and traditional system versus various values of $\beta$. It is shown in figure that the ergodic sum rate of both systems does not change significantly for low values of RSI (i.e. $\beta=-50 \mathrm{~dB}$ to $\beta \approx-10 \mathrm{~dB}$ ), but it starts to change at high values of RSI (i.e. $\beta=-10 \mathrm{~dB}$ to $\beta \approx-1$ $\mathrm{dB})$.

However, the performance of the proposed system remains higher than traditional system in terms of ergodic sum rate under various levels of RSI. Therefore, the proposed ASIC based FD NOMA system shows better ergodic sum rate than traditional SIC based FD NOMA system for various values of RSI.

\section{Conclusions}

This paper has proposed FD DF cooperative NOMA system, where a novel ASIC scheme is introduced in the system that makes the proposed system more effective in practice as compared to traditional SIC based FD DF cooperative NOMA system. The approximated analytical expressions of outage probability of proposed ASIC based FD DF cooperative NOMA system are derived and investigated under realistic scenario of RSI. In addition, the proposed ASIC based FD DF cooperative NOMA system is also analysed by approximating the expressions of ergodic sum rate. The proposed ASIC based FD DF cooperative NOMA system is found to be superior in outage performance and ergodic sum rate than traditional SIC based FD DF cooperative NOMA system in medium to high SNR range and achieved the large system throughput under same conditions. This work is primarily concerned with single input and single output (SISO) FD DF cooperative NOMA system but it can be extended into multiple input and multiple output (MIMO) FD DF cooperative NOMA system in future.

\section{References}

[1] DAI, L., WANG, B., YUAN, Y., et al. Non-orthogonal multiple access for $5 \mathrm{G}$ : solutions, challenges, opportunities, and future research trends. IEEE Communications Magazine, 2015, vol. 53, no. 9, p. 74-81. DOI: 10.1109/MCOM.2015.7263349

[2] SHIMOJO, T., UMESH, A., FUJISHIMA, A., et al. Special articles on $5 \mathrm{G}$ technologies toward 2020 deployment. NTT DOCOMO Technologies, 2016, vol. 17, no. 4, p. 50-59.

[3] SAITO, Y., KISHIYAMA, Y., BENJEBBOUR, A., et al. Nonorthogonal multiple access for cellular future radio access. In IEEE Vehicular Technology Conference. Dresden (Germany), 2013, p. 1-5. DOI: 10.1109/VTCSpring.2013.6692652

[4] SAITO, K., BENJEBBOUR, A., KISHIYAMA, Y., et al. Performance and design of SIC receiver for downlink NOMA with open-loop SU-MIMO. In IEEE International Conference on Communication Networks. London (UK), 2015. DOI: 10.1109/ICCW.2015.7247334

[5] WEI, Z., YUAN, J., Ding, Z., et al. A survey of downlink nonorthogonal multiple access for $5 \mathrm{G}$ wireless communication networks. ZTE Communication, 2016, vol. 14, no. 4, p. 17-23.

[6] DING, Z., LIE, X., KARAGIANNIDIS, K., et al. A survey on non-orthogonal multiple access for $5 \mathrm{G}$ networks: Research challenges and future trends. IEEE Journal on Selected Areas in Communications, 2017, vol. 35, no. 10, p. 2181-2195. DOI: 10.1109/JSAC.2017.2725519

[7] ZHANG, S., XU, X., LU, L., et al. Sparse code multiple access: An energy efficient uplink approach for $5 \mathrm{G}$ wireless systems. In IEEE Global Communication Conference. Austin (USA), 2014. DOI: 10.1109/GLOCOM.2014.7037563

[8] DING, Z., LIU, Y., CHOI, J., et al. Application of non-orthogonal multiple access in LTE and $5 \mathrm{G}$ networks. IEEE Communications Magazine, 2017, vol. 55, no. 2, p. 185-191. DOI: 10.1109/MCOM.2017.1500657CM

[9] OTAO, N., KISHIYAMA, Y., HIGUCHI, K. Performance of nonorthogonal multiple access with SIC in cellular downlink using proportional fair based resource allocation. IEICE Transactions on Communications, 2015, vol. 98, no. 2, p. 344-351. DOI: 10.1109/ISWCS.2012.6328413

[10] USMAN, R., KHAN, A., USMAN, A., et al. On the performance of perfect and imperfect SIC in downlink non orthogonal multiple access. In International Conference on Smart Green Technology in Electrical and Information Systems, 2016. DOI: 10.1109/ICSGTEIS.2016.7885774

[11] IMARI, A., XIAO, P., IMRAN, A., et al. Uplink non-orthogonal multiple access for 5G wireless networks. In International Symposium on Wireless Communications Systems. Barcelona (Spain), 2014. DOI: 10.1109/ISWCS.2014.6933459

[12] ZHANG, N., WANG, J., KANG, J.,et al. Uplink non-orthogonal multiple access in 5G systems. IEEE Communication Letters, 2016, vol. 20, no. 3, p. 458-461. DOI: 10.1109/LCOMM.2016.2521374 
[13] DING, Z., YANG, Z., FAN, P., et al. On the performance of non-orthogonal multiple access in $5 \mathrm{G}$ systems with randomly deployed users. IEEE Signal Processing Letters, 2014, vol. 21, no. 12, p. 1501-1505. DOI: 10.1109/LSP.2014.2343971

[14] DING, Z., FAN, P., POOR, V. Impact of user pairing on 5G nonorthogonal multiple-access downlink transmissions. IEEE Transactions on Vehicular Technology, 2016, vol. 65, no. 8, p. 6010-6023. DOI: $10.1109 /$ TVT.2015.2480766

[15] LIU, X., WANG, X., LIU, Y. Power allocation and performance analysis of the collaborative NOMA assisted relaying systems in 5G. China Communications, 2017, vol. 14, no. 1, p. 50-60. DOI: 10.1109/CC.2017.7839757

[16] OZDURAN, V. Advanced successive interference cancellation for non-orthogonal multiple access. In 26th Telecommunications Forum. Belgrade (Serbia), 2018, p. 1-4. DOI: 10.1109/TELFOR.2018.8612111

[17] MEN, J., GE, J. Non-orthogonal multiple access for multiple-antenna relaying networks. IEEE Communication Letters, 2015, vol. 19, no. 10, p. 1686-1689. DOI: 10.1109/LCOMM.2015.2472006

[18] KIM, J., LEE, I. Non-orthogonal multiple access in coordinated direct and relay transmission. IEEE Communication Letters, 2015, vol. 19, no. 11, p. 2037-2040. DOI: 10.1109/LCOMM.2015.2474856

[19] DING, Z., PENG, M., POOR, V. Cooperative non-orthogonal multiple access in 5G systems. IEEE Communication Letters, 2015, vol. 19, no. 8, p. 1462-1465. DOI: 10.1109/LCOMM.2015.2441064

[20] JU. H., OH, E., HONG, D. Improving efficiency of resource usage in two-hop full duplex relay systems based on resource sharing and interference cancellation. IEEE Transactions on Wireless Communications, 2009, vol. 8, no. 8, p. 3933-3938. DOI: 10.1109/TWC.2009.081049

[21] DUARTE, M., DICK, C., SABHARWAL, A. Experiment-driven characterization of full-duplex wireless systems. IEEE Transactions on Wireless Communications, 2012, vol. 11, no. 12, p. 4296-4307. DOI: 10.1109/TWC.2012.102612.111278

[22] JIMENEZ, L., RODRIQUEZ, I., TRAN, N., et al. Performance of full duplex AF relaying in the presence of residual self-interference. IEEE Journal on Selected Areas in Communications, 2014, vol. 32, no. 9, p. 1752-1764. DOI: 10.1109/JSAC.2014.2330151

[23] RODRIGUEZ, L., TRAN, N., LE-NQOC, T. Optimal power allocation and capacity of full-duplex AF relaying under residual selfinterference. IEEE Wireless Communication Letters, 2014, vol. 3, no. 2, p. 233-236. DOI: 10.1109/WCL.2014.020614.130831

[24] KWON, T., LIM, S., CHOI, S., et al. Optimal duplex mode for DF relay in terms of the outage probability. IEEE Transactions on Vehicular Technology, 2010, vol. 59, no. 7, p. 3628-3634. DOI: $10.1109 /$ TVT.2010.2050503

[25] SOHAIB, S., UPPAL, M. Full duplex compress-and-forward relaying under residual self interference. IEEE Transactions on Vehicular Technology, 2018, vol. 67, no. 3, p. 2776-2780. DOI: 10.1109/LCOMM.2016.2611500

[26] ZHANG, Z., MA, Z., XIAO, M., et al. Full-duplex device-to device aided cooperative non-orthogonal multiple access. IEEE Transactions on Vehicular Technology, 2017, vol. 66, no. 5, p. 4467-4471. DOI: 10.1109/TVT.2016.2600102

[27] MEN, J., GE, J. Performance analysis of non-orthogonal multiple access in downlink cooperative network. IET Communications, 2015 , vol. 9, no. 18, p. 2267-2273. DOI: 10.1049/iet-com.2015.0203

[28] ZHONG, C., ZHANG, Z. Non-orthogonal multiple access with cooperative full-duplex relaying. IEEE Communication Letters, 2016, vol. 20, no. 12, p. 2478-2481. DOI: 10.1109/LCOMM.2016.2611500
[29] GRADSHTEYN, I., RYZHIK, I. Table of Integrals, Series and Products. 6th ed., New York (USA): Academic Press, 2000. ISBN: 9780080542225

[30] HILDEBRAND, F. Introduction to Numerical Analysis. 2nd ed., Mineola. New York (USA): Dover Publications, 1987. ISBN: 9780486653631

\section{About the Authors...}

Qaiser HUSSAIN received his BE degree in Electronics Engineering from NED University of Engineering and Technology, Karachi, Pakistan in 2002. He worked as research associate at National University Science and Technology (NUST) in 2002 and then received his MS degree in Electrical Engineering from University of Engineering and Technology (UET), Taxila, Pakistan in 2010. Currently, he is a PhD scholar and working as Lecturer in the Department of Electrical Engineering of Riphah International University, Islamabad, Pakistan. His research interests include full duplex wireless communication, wireless sensor networks and cooperative MIMO communication.

Sarmad SOHAIB received BS in Electronics Engineering from Ghulam Ishaq Khan Institute of Engineering Sciences and Technology, Pakistan, in 2005. He received his PhD degree in Electrical Engineering from the University of Manchester, United Kingdom in 2010. Currently, he is working as an Associate Professor at University of Engineering and Technology, Taxila, Pakistan and University of Jeddah, KSA. His research interests include energy efficient wireless communication, wireless sensor networks and full duplex cooperative wireless communication.

\section{Appendix A: Proof of Proposition 1}

The outage probability of far user $D_{1}$ is given as [28]

$$
\begin{aligned}
& P_{D 1}^{F D}=1-P\left(C_{\mathrm{r}, 1}>R_{1} \quad C_{1,1}>R_{1} \quad C_{1,3}>R_{3}\right) \\
& =1-P\left(\frac{a_{1} P\left|h_{\mathrm{sr}}\right|^{2}}{\frac{V}{2}+\frac{N_{0}}{2}}>\gamma_{\mathrm{th} 1} \frac{c_{1} P\left|h_{\mathrm{r} 1}\right|^{2}}{\frac{N_{0}}{2}}>\gamma_{\mathrm{th} 1}\right. \\
& \left.\frac{c_{3} P\left|h_{\mathrm{r} 1}\right|^{2}}{c_{1} P\left|h_{\mathrm{r} 1}\right|^{2}+\frac{N_{0}}{2}}>\gamma_{\mathrm{th} 3}\right) \\
& =1-P\left(\frac{a_{1} \gamma_{\mathrm{sr}}}{\frac{V}{2 N_{0}}+\frac{1}{2}}>\gamma_{\mathrm{th} 1} 2 c_{1} \gamma_{\mathrm{r} 1}>\gamma_{\mathrm{th} 1}\right. \\
& \left.\frac{c_{3} \gamma_{\mathrm{r} 1}}{c_{1} \gamma_{\mathrm{r} 1}+\frac{1}{2}}>\gamma_{\mathrm{th} 3}\right) \\
& =1-P\left(\gamma_{\mathrm{sr}}>\frac{\gamma_{\mathrm{th} 1}\left(V+N_{0}\right)}{2 a_{1}} \gamma_{\mathrm{r} 1}>\frac{\gamma_{\mathrm{th} 1}}{2 c_{1}}\right. \\
& \left.\gamma_{\mathrm{r} 1}>\frac{\gamma_{\mathrm{th} 3}}{2\left(c_{3}-c_{1} \gamma_{\mathrm{th} 3}\right)}\right)
\end{aligned}
$$


where the variables $\gamma_{\mathrm{sr}}$ and $\gamma_{\mathrm{r} 1}$ have exponential distribution with parameters $\rho_{\mathrm{sr}}^{\gamma}$ and $\rho_{\mathrm{r} 1}{ }^{\gamma}$ respectively. Therefore,

$$
\begin{aligned}
P_{D 1}^{F D} & =1-\mathrm{e}^{-\left[\frac{\gamma_{\mathrm{th} 1}\left(V+N_{0}\right)}{2 a_{1} \rho_{\mathrm{sr}} \gamma}+\frac{\gamma_{\mathrm{h} 1}}{2 c_{1} \rho_{\mathrm{r}_{1}} \gamma}+\frac{\gamma_{\mathrm{th} 3}}{2\left(c_{3}-c_{1} \gamma_{\mathrm{h} 3}\right) \rho_{\mathrm{r} 1}{ }^{\gamma}}\right]} \\
& =1-\mathrm{e}^{-\theta_{1}}
\end{aligned}
$$

where $\theta_{1}=\left[\frac{\gamma_{\mathrm{th} 1}\left(V+N_{0}\right)}{2 a_{1} \rho_{\mathrm{sr}} \gamma}+\frac{\gamma_{\mathrm{th} 1}}{2 c_{1} \rho_{\mathrm{r} 1} \gamma}+\frac{\gamma_{\mathrm{th} 3}}{2\left(c_{3}-c_{1} \gamma_{\mathrm{th} 3}\right) \rho_{\mathrm{r} 1} \gamma}\right]$

\section{Appendix B: Proof of Proposition 2}

The outage probability of far user $D_{2}$ is written as [28]

$$
\begin{aligned}
& P_{D 2}^{F D}=1-P\left(C_{\mathrm{r}, 2}>R_{2} \quad C_{2,2}>R_{2}\right) \\
& =1-P\left(\frac{2 c_{2} P\left|h_{\mathrm{r} 2}\right|^{2}}{N_{0}}>\gamma_{\mathrm{th} 2} \frac{a_{2} P\left|h_{\mathrm{sr}}\right|^{2}}{\frac{V}{2}+\frac{N_{0}}{2}}>\gamma_{\mathrm{th} 2}\right) \\
& =1-P\left(2 c_{2} \gamma_{\mathrm{r} 2}>\gamma_{\mathrm{th} 2} \frac{a_{2} \gamma_{\mathrm{sr}}}{\frac{V}{2 N_{0}}+\frac{1}{2}}>\gamma_{\mathrm{th} 2}\right) \\
& =1-P\left(\gamma_{\mathrm{r} 2}>\frac{\gamma_{\mathrm{th} 2}}{2 c_{2}} \quad \gamma_{\mathrm{sr}}>\frac{\gamma_{\mathrm{th} 2}\left(V+N_{0}\right)}{2 a_{2}}\right) \\
& =1-\mathrm{e}^{-\frac{\gamma_{\mathrm{th} 2}}{2 c_{2} \rho_{\mathrm{r} 2} \gamma}} \times e^{-\frac{\gamma_{\mathrm{th} 2}\left(V+N_{0}\right)}{2 a_{2} \rho_{\mathrm{sr}} \gamma}} \\
& =1-\mathrm{e}^{-\frac{\gamma_{\mathrm{th} 2}}{2}\left[\frac{1}{c_{2} \rho_{\mathrm{r} 2}^{\gamma}}+\frac{V+N_{0}}{a_{2} \rho_{\mathrm{sr}} \gamma}\right]} \\
& =1-\mathrm{e}^{-\frac{\gamma_{\mathrm{th} 2} \theta_{2}}{2}}
\end{aligned}
$$

where $\theta_{2}=\left(\phi_{1}+\phi_{2}\right), \phi_{1}=\frac{1}{c_{2} \rho_{\mathrm{r} 2} \gamma}$, and $\phi_{2}=\frac{V+N_{0}}{a_{2} \rho_{\mathrm{sr}} \gamma}$.

\section{Appendix C: Proof of Proposition 3}

The outage probability of far user $D_{3}$ for evaluating the result of (23) can be proceeded as

$$
\begin{aligned}
P_{D 3}^{F D} & =1-P\left(\frac{c_{3} \gamma_{\mathrm{r} 3}}{c_{1} \gamma_{\mathrm{r} 3}+\frac{1}{2}}>\gamma_{\mathrm{th} 3}\right. \\
& =1-P\left(\gamma_{\mathrm{r} 3}>\frac{a_{3} \gamma_{\mathrm{sr}}}{a_{1} \gamma_{\mathrm{sr}}+\frac{V}{2 N_{0}}+\frac{1}{2}}>\gamma_{\mathrm{th} 3}\right) \\
& =1-\left[\mathrm{e}^{-\frac{\left.\gamma_{\mathrm{th} 3}-c_{2} \gamma_{\mathrm{th} 3}\right)}{2\left(c_{3}-c_{1} \gamma_{\mathrm{th} 3}\right) \rho_{\mathrm{r} 3} \gamma}}\right. \\
& \left.\gamma_{\mathrm{sr}}>\frac{\gamma_{\mathrm{th} 3}\left(V+N_{0}\right)}{2\left(a_{3}-a_{1} \gamma_{\mathrm{th} 3}\right)}\right) \\
& \left.=1-\mathrm{e}^{-\frac{\gamma_{\mathrm{th} 3}}{2}\left[\frac{1}{\left(c_{3}-c_{1} \gamma_{\mathrm{th} 3}\right) \rho_{\mathrm{r} 3} \gamma}+\frac{V+N_{0}}{\left(a_{3}-a_{1} \gamma_{\mathrm{th} 3}\right) \rho_{\mathrm{sr}} \gamma}\right.}\right] \\
& =1-\mathrm{e}^{-\frac{\gamma_{\mathrm{th} 3} \theta_{3}}{2}}
\end{aligned}
$$

where $\theta_{3}=\left(\psi_{1}+\psi_{2}\right), \psi_{1}=\frac{1}{\left(c_{3}-c_{1} \gamma_{\mathrm{th} 3}\right) \rho_{\mathrm{r} 3} \gamma}$, and $\psi_{2}=$ $\frac{V+N_{0}}{\left(a_{3}-a_{1} \gamma_{\mathrm{th} 3}\right) \rho_{\mathrm{sr}} \gamma}$.

\section{Appendix D: Proof of Proposition 4}

According to the rate of far user $D_{1}$ given by (18), we assume $X=\min \left(\frac{a_{1} \gamma_{\mathrm{sr}}}{\frac{V}{2}+\frac{1}{2}}, 2 c_{1} \gamma_{\mathrm{r} 1}\right)$. The CDF of $X$ is given by

$$
\begin{aligned}
F_{\mathrm{X}}(x) & =P\left(\min \left(\frac{a_{1} \gamma_{\mathrm{sr}}}{\frac{V}{2}+\frac{1}{2}}, 2 c_{1} \gamma_{\mathrm{r} 1}\right)<x\right) \\
& =1-P\left(\gamma_{\mathrm{sr}}>\frac{x(V+1)}{2 a_{1}}\right) P\left(\gamma_{\mathrm{r} 1}>\frac{x}{2 c_{1}}\right) \\
& =1-\left(\mathrm{e}^{-\frac{x(V+1)}{2 a_{1} \rho_{\mathrm{sr}} \gamma}} \times \mathrm{e}^{-\frac{x}{2 c_{1} \rho_{\mathrm{r} 1} \gamma}}\right) .
\end{aligned}
$$

Now, by using the following identity [28]

$$
\int_{0}^{\infty} \log _{2}(1+x) f_{X}(x) \mathrm{d} x=\frac{1}{\ln 2} \int_{0}^{\infty} \frac{1-\mathrm{F}_{X}(x)}{1+x} \mathrm{~d} x .
$$

$\mathrm{E}\left\{C_{1}\right\}$ could be calculated [28] as

$$
\begin{aligned}
\mathrm{E}\left\{C_{1}\right\} & =\mathrm{E}\left\{\log _{2}\left(1+\min \left(\frac{a_{1} \gamma_{\mathrm{sr}}}{\frac{V}{2}+\frac{1}{2}}, 2 c_{1} \gamma_{\mathrm{r} 1}\right)\right\}\right. \\
& =\frac{1}{\ln 2} \int_{0}^{\infty} \frac{\mathrm{e}^{-\frac{x(V+1)}{2 a_{1} \rho_{\mathrm{sr}} \gamma}} \times \mathrm{e}^{-\frac{x}{2 c_{1} \rho_{\mathrm{r} 1} \gamma}}}{1+x} \mathrm{~d} x \\
& =\frac{1}{\ln 2} \int_{0}^{\infty} \frac{\mathrm{e}^{-x\left[\frac{(V+1)}{2 a_{1} \rho_{\mathrm{sr}} \gamma}+\frac{1}{2 c_{1} \rho_{\mathrm{r} 1} \gamma}\right]}}{1+x} \mathrm{~d} x .
\end{aligned}
$$

Now by substituting $(x+1)\left[\frac{(V+1)}{2 a_{1} \rho_{\mathrm{sr}} \gamma}+\frac{1}{2 c_{1} \rho_{\mathrm{r} 1} \gamma}\right]=u$ in (D3), we get

$$
\mathrm{E}\left\{C_{1}\right\}=\frac{\mathrm{e}^{\left(\frac{V+1}{2 a_{1} \rho_{\mathrm{sr}} \gamma}+\frac{1}{2 c_{1} \rho_{\mathrm{r} 1} \gamma}\right)}}{\ln 2} \times \int_{\left(\frac{V+1}{2 a_{1} \rho_{\mathrm{sr}} \gamma}+\frac{1}{2 c_{1} \rho_{\mathrm{r} 1} \gamma}\right)}^{\infty} \frac{\mathrm{e}^{-u}}{u} \mathrm{~d} u
$$

Finally, by using the definition of exponential integral which is given as $\mathrm{E}_{1}(x)=\int_{x}^{\infty} \frac{\mathrm{e}^{-u}}{u} \mathrm{~d} u$, and it is also given in [29], Eq. 3.351.5. The above equation becomes

$$
\mathrm{E}\left\{C_{1}\right\}=\frac{\mathrm{e}^{\left(\frac{V+1}{2 a_{1} \rho_{\mathrm{sr}} \gamma}+\frac{1}{2 c_{1} \rho_{\mathrm{r} 1} \gamma}\right)}}{\ln 2} \times \mathrm{E}_{1}\left(\frac{V+1}{2 a_{1} \rho_{\mathrm{sr}} \gamma}+\frac{1}{2 c_{1} \rho_{\mathrm{r} 1} \gamma}\right) .
$$




\section{Appendix E: Proof of Proposition 5}

The ergodic rate of far user $D_{2}$ for deriving the result of (26) is expressed as

$$
\mathrm{E}\left\{C_{2}\right\}=E\left[\log _{2}\left(1+\min \left\{\gamma_{\mathrm{r}, 2}, \gamma_{2,2}\right\}\right)\right] .
$$

Here we assume $Y=\min \left(\gamma_{\mathrm{r}, 2}, \gamma_{2,2}\right)$. Its $\mathrm{CDF}$ is

$$
\begin{aligned}
\mathrm{F}_{Y}(y) & =P\left(\min \left(\gamma_{\mathrm{r}, 2}, \gamma_{2,2}\right)<y\right) \\
& =1-P\left(\frac{2 a_{2} \gamma_{\mathrm{sr}}}{V+1}>y\right) \times P\left(\left(2 c_{2} \gamma_{\mathrm{r} 2}\right)>y\right) \\
& =1-P\left(\gamma_{\mathrm{sr}}>\frac{y(V+1)}{2 a_{2}}\right) \times P\left(\gamma_{\mathrm{r} 2}>\frac{y}{2 c_{2}}\right) \\
& =1-\mathrm{e}^{-\frac{y(V+1)}{2 a_{2} \rho_{\mathrm{sr}} \gamma}} \times \mathrm{e}^{-\frac{y}{c_{2} \rho_{\mathrm{r}} \gamma}} \\
& =1-\mathrm{e}^{-y\left[\frac{V+1}{2 a_{2} \rho_{\mathrm{sr}} \gamma}+\frac{1}{2 c_{2} \rho_{\mathrm{r} 2} \gamma}\right] .}
\end{aligned}
$$

The ergodic rate of far user $D_{2}$ can be calculated as

$$
\mathrm{E}\left\{C_{2}\right\}=\frac{1}{\ln 2} \int_{0}^{\infty} \frac{\mathrm{e}^{-y\left[\frac{V+1}{2 a_{2} \rho_{\mathrm{sr}} \gamma}+\frac{1}{2 c_{2} \rho_{\mathrm{r} 2} \gamma}\right]}}{1+y} \mathrm{~d} y .
$$

Here by using the definition of exponential integral, (E3) can be simplified as

$$
\begin{aligned}
& \mathrm{E}\left\{C_{2}\right\}=\frac{\mathrm{e}^{\left(\frac{V+1}{2 a_{2} \rho_{\mathrm{sr}} \gamma}+\frac{1}{2 c_{2} \rho_{\mathrm{r}} \gamma}\right)}}{\ln 2} \\
& \times\left[\mathrm{E}_{1}\left(\frac{V+1}{2 a_{2} \rho_{\mathrm{sr}} \gamma}+\frac{1}{2 c_{2} \rho_{\mathrm{r} 2} \gamma}\right)\right] .
\end{aligned}
$$

\section{Appendix F: Proof of Proposition 6}

The ergodic rate of far user $D_{3}$ can be expressed as:

$$
\mathrm{E}\left\{\mathrm{r}_{3}\right\}=\mathrm{E}\left[\log _{2}\left(1+\min \left\{\gamma_{\mathrm{r}, 3}, \gamma_{1,3}, \gamma_{3,3}\right\}\right)\right] \text {. }
$$

Here we suppose $\left.Z=\min \left(\gamma_{\mathrm{r}, 3}, \gamma_{1,3}, \gamma_{3,3}\right\}\right)$. The CDF of $Z$ is calculated as follows

$$
\begin{aligned}
& \mathrm{F}_{Z}(z)=P\left(\operatorname { m i n } \left(\frac{a_{3} \gamma_{\mathrm{sr}}}{a_{1} \gamma_{\mathrm{sr}}+\frac{V}{2}+\frac{1}{2}}, \frac{c_{3} \gamma_{\mathrm{r} 1}}{c_{1} \gamma_{\mathrm{r} 1}+\frac{1}{2}},\right.\right. \\
&\left.\left.\frac{c_{3} \gamma_{\mathrm{r} 3}}{c_{1} \gamma_{\mathrm{r} 3}+\frac{1}{2}}\right)<z\right) \\
&=1-P\left(\operatorname { m i n } \left(\frac{a_{3} \gamma_{\mathrm{sr}}}{a_{1} \gamma_{\mathrm{sr}}+\frac{V}{2}+\frac{1}{2}}, \frac{c_{3} \gamma_{\mathrm{r} 1}}{c_{1} \gamma_{\mathrm{r} 1}+\frac{1}{2}},\right.\right. \\
&\left.\left.\frac{c_{3} \gamma_{\mathrm{r} 3}}{c_{1} \gamma_{\mathrm{r} 3}+\frac{1}{2}}\right)>z\right) \\
&=1-\left[\mathrm{e}^{-\frac{z(V+1)}{2\left(a_{3}-z a_{1}\right) \rho_{\mathrm{sr}} \gamma}} \times \mathrm{e}^{-\frac{z}{2\left(c_{3}-z c_{1}\right) \rho_{\mathrm{r} 1} \gamma}}\right. \\
&\left.\times \mathrm{e}^{-\frac{z}{2\left(c_{3}-z c_{1}\right) \rho_{\mathrm{r} 3} \gamma}}\right] .
\end{aligned}
$$

Using $\mathrm{F}_{Z}(z)$, the $\mathrm{E}\left\{C_{3}\right\}$ is

$$
\mathrm{E}\left\{C_{3}\right\}=\frac{1}{\ln 2} \int_{0}^{\frac{a_{3}}{a_{1}}} \frac{\mathrm{e}^{-z t}}{(1+z)} \mathrm{d} z
$$

where

$t=\left(\frac{V+1}{2\left(a_{3}-z a_{1}\right) \rho_{\mathrm{sr}} \gamma}+\frac{1}{2\left(c_{3}-z c_{1}\right) \rho_{\mathrm{r} 1} \gamma}+\frac{1}{2\left(c_{3}-z c_{1}\right) \rho_{\mathrm{r} 3} \gamma}\right)$.

The Gaussian Quadrature method [30] is used to simplify the integral (F.3), and using the approximation as:

$$
\int_{a}^{b} g(x) \mathrm{d} x=\frac{b-a}{2} \sum_{i=1}^{N} w_{i} g\left(\left(\frac{b-a}{2}\right) X_{i}+\left(\frac{b+a}{2}\right)\right) .
$$

Now by assuming the following function of $g(x)$

$$
g(x)=\frac{\mathrm{e}^{-z\left(\frac{V+1}{2\left(a_{3}-z a_{1}\right) \rho_{\mathrm{s}} \gamma}+\frac{1}{2\left(c_{3}-z c_{1}\right) \rho_{\mathrm{r}} \gamma^{\gamma}}+\frac{1}{2\left(c_{3}-z c_{1}\right) \rho_{\mathrm{r}} \gamma}\right)}}{(1+z)} .
$$

And using (F4), we can derive the desired result of (27). 